\title{
Tip-over stability analysis of orchard vehicle with dynamic simulation method
}

\author{
Weiwei $\mathrm{Li}^{1}$, Lintao Tian $^{2}$, Shaohong Zhang ${ }^{3}$, Shumao Wang ${ }^{4}$, Xin Wang ${ }^{5, a}$ \\ Beijing Key Laboratory of Optimized Design for Modern Agricultural Equipment \\ ${ }^{1-5}$ College of Engineering, China Agricultural University, Beijing, 10083, China \\ aemail: wangxin117@cau.edu.cn
}

Keywords: orchard vehicle; driving stability control model; ADAMS; Matlab; joint simulation

\begin{abstract}
This paper is focused on orchard vehicle rollover and tipping problems in its operation process. Firstly, seven degrees of freedom orchard vehicle ADAMS model is established. And then, it built up a driving stability control algorithm to adjust input speed by real-time pitch angle, roll angle and other state parameters. After that, ADAMS and MATLAB joint simulation control model is setup for doing simulation experiments with which results can be used to adjust the coefficients of control model. Finally, it also established physical scale model at the same time to verify simulation results. The experimental results show that orchard vehicle control driving stability model, which proposed in this paper, can effectively prevent the orchard vehicle tilting, and can improve the driving stability.
\end{abstract}

\section{Introduction}

With development of orchard industry, machinery demand of fruit growers is becoming more and stronger. Orchard vehicle can meet pruning, picking, transportation and a set of functions which needed in orchard environment. So orchard vehicle became more and more useful and popular in orchard operation. However, accidents usually happen during operation of orchard vehicle, causing property damage and threaten drivers' safety ${ }^{[1]}$. Accident happened for two reasons: First, orchard working environment is much complex than normal road ${ }^{[2]}$; Second, center mass of orchard vehicle is on high side which makes tilting and rollover accidents happen easily. So the research of orchard vehicle rollover and tipping prevention shows great significance for orchard operation and safety.

At present, a lot research of vehicle tilting prevention is concentrated on heavy-duty vehicles and early warning mechanism. Chen $\mathrm{B}^{[1]}$ launched crane tilting prevention method based on luffing cylinder hydraulic truck; Seung-Bok Choi ${ }^{[3]}$ studied the orchard locomotive anti-collision tilting prevention system based on ZigBee. Preston Thomas ${ }^{[2]}$ has developed a set of stability control and warning system in the heavy vehicle. Orchard vehicle rollover and tipping problems is different from heavy vehicles, therefore, this article is mainly about orchard vehicle active rollover and tipping prevention control method research, to suitable for rough and changeable working environment of orchard vehicle.

\section{Orchard vehicle model}

While orchard vehicle is driven on irregular roads, the center of gravity, pitching angle and its acceleration, roll angle and its acceleration are changing with fluctuation of road. To study these parameters can make effort on controlling stability of orchard vehicle. Assume vehicle body is a rigid body, seven degrees of freedom dynamic model is established, which is shown in figure 1. 


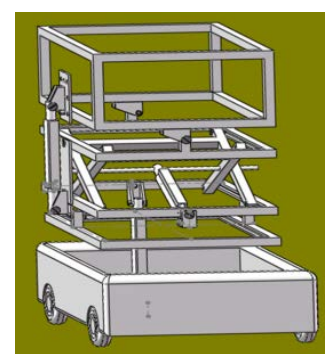

Fig.1. Orhard vehicle 1:3 Scaled ADAMS model

\subsection{Simulation model}

ADAMS (Automatic Dynamic Analysis of Mechanical Systems), virtual prototype Analysis software, developed by American MDI application, is very widely used ${ }^{[4]}$. Using ADAMS can easily do analysis about statics, kinematics and dynamics of virtual prototype.

According to dynamic model with seven degrees of freedom and scale model ${ }^{[5-7]}$, we established the virtual prototype simulation model, which is mainly composed of body, tires, power, etc. Modeling process is as follows: (1) We established basic component of the scale model in the ADAMS/VIEWS; (2) Confirmed the topology of the components and combined the components. To determine the quality of the components, parameters such as center of mass and moment of inertia of the coordinate system according to the parameters of table 1; (3) Determined the connection position constraint relation of parts, and add all kinds of constraints in turn according to the component movements; (4) The parameters were parameterized definition. The design accuracy of the simulation model has an important influence to the control effect. Therefore, in order to ensure the accuracy of the virtual prototype model, the simulation model should be for further analysis, check and correction after completed.

Different kind of tire model in different environment can affect the calculation validity the accuracy; therefore it is very important to choose the right model. In vehicle stability control test, we adopt the UA tire model, based on the magic formula, embedded in the ADAMS. General expression of the magic formula is:

$$
Y(x) D \sin \left\{\operatorname{Ctan}^{-1}\left[B x-E\left(B x-\tan ^{-1}(B x)\right)\right]\right\}
$$

where, $Y(x)$ is lateral force, back to the positive moment or longitudinal force, $X$ is tire side-slip Angle or longitudinal slip ratio, $B, D, E$ is coefficient, determined by the vertical Angle and camber Angle of the tire, $C$ is curve shape factor.

UA tire has good robustness, and the model considers the unsteady effect. It is analyzed through the friction circle of cornering and longitudinal sliding interaction, as well as extraversion and relaxation length and radius of the tire. The concise mathematical model and the accuracy is high, therefore, this paper selects the UA tire in virtual prototype model.

\subsection{Scaled model}

As the orchard vehicle' tilting real test is dangerous and expensive, so we established a 1:5 scale model in lab. Firstly, we established a virtual prototype of the scale model in ADAMs. And some tests were done to analysis the stability while vehicles were driven under the condition of simulated orchard road. Then we used MATLAB/Simulink module to do joint simulation, debugging various parameters of the control model, to optimize our control method. The main parameters scale model is shown in table 1.

Table 1 Scale model parameters

\begin{tabular}{cc|cc}
\hline Name & Value & Name & Value \\
\hline $\begin{array}{c}\text { acceleration } \\
\text { of gravity }\end{array}$ & $9.81 \mathrm{~m} / \mathrm{s} 2$ & $\begin{array}{c}\text { Moment of } \\
\text { inertia around the Z axis }\end{array}$ & $\begin{array}{c}0.0132 \\
\mathrm{~kg} \cdot \mathrm{m} 2\end{array}$ \\
\hline Body quality & $2.04 \mathrm{~kg}$ & Each wheel weight & $32.36 \mathrm{~g}$ \\
\hline Body length & $0.206 \mathrm{~m}$ & Wheel radius & $32.5 \mathrm{~mm}$ \\
\hline Body width & $0.156 \mathrm{~m}$ & Wheel width & $25.0 \mathrm{~mm}$ \\
\hline
\end{tabular}




\begin{tabular}{ccc|cc} 
Body height & $0.152 \mathrm{~m}$ & Wheel axis moment of inertia & $2.5 \times 10-5 \mathrm{~kg} \cdot \mathrm{m} 2$ \\
\hline $\begin{array}{c}\text { Moment of inertia } \\
\text { around the X axis }\end{array}$ & $0.0081 \mathrm{~kg} \cdot \mathrm{m} 2$ & Wheel yaw moment of inertia & $1.5 \times 10-5 \mathrm{~kg} \cdot \mathrm{m} 2$ \\
\hline $\begin{array}{c}\text { Moment of inertia } \\
\text { around the Y axis }\end{array}$ & $0.0100 \mathrm{~kg} \cdot \mathrm{m} 2$ & Total mass & $2.17 \mathrm{~kg}$ \\
\hline
\end{tabular}

\subsection{Driving stability control model}

When orchard vehicle go through obstacles, the most direct manifestation of the tilting is that the contact force between the wheel and the ground state is zero. So the contact force $u$ should satisfy

$$
|u| \geq 0
$$

Vehicle tilting problem is mainly composed of body pitching Angle to describe, so while judging the acceleration, we can regard pitching Angle parameters as the control aim. In concrete implementation, we can use the sensor to measure the change of the pitch Angle. In order to brake signals to wheels, we also need to grasp the vehicle's other status parameters, including pitch body acceleration, the pitch angular velocity and the direction. Then we make the wheels with speed $v$

$$
v=K_{p} \varphi+K_{d} \dot{\varphi}
$$

$K_{p}, K_{d^{-}}$adjustment coefficient.

The vehicles' tilting can be simplified as a process of uphill and downhill. When vehicles are uphill, pitching Angle increases gradually, and pitching Angle velocity changes. Contact force between wheels and the surface changes. When pitching Angle increases to a certain degree, the front wheel no longer meet the formula (2), vehicles will tilt to the rear; when vehicles are downhill, pitching Angle gradually decreases, and when pitching Angle decreases to a certain degree, the rear wheel no longer meet the formula (2) type, vehicle will tilt to the front. After adding control from formula (3), the vehicle braking force changes on the basis of pitching Angle and pitching Angle velocity. Through the way of reducing or increasing the speed to enhance the adhesive ability between tire and road surface, so to improve the stability and prevent tilting.

\section{Simulation experiments}

\subsection{Simulation experiment and result analysis}

Different happens when orchard vehicle driven ion different roads, we did some simple simulation experiments in ADAMS to select proper pavement parameters. Then we used this kind of model to do joint simulation test using ADAMS and MATLAB.

Irregular surfaces can be regarded as composed of a large number of uniform and small waves; therefore, the vehicles' motion in simulation can be simplified as movement under the fixed fluctuation frequency and irregular ups and downs road. 2d sinusoidal road in ADAMS was used in this paper, including three types of road. The amplitude is $30 \mathrm{~mm}$ and wavelength is $200 \mathrm{~mm}, 400$ $\mathrm{mm}$ and $600 \mathrm{~mm}$ respectively. Pitching Angle changed with time curve as shown in figure 2.

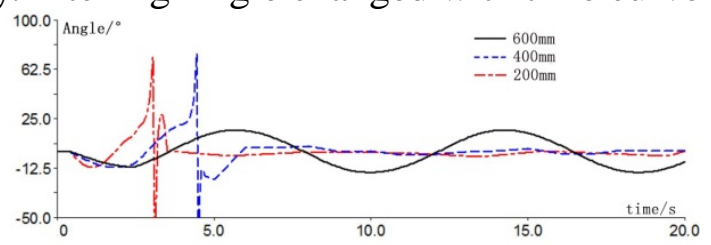

Figure.2. ADMAS simulation test results

The result shows that when the road surface fluctuation is small, such as wavelength $200 \mathrm{~mm}$ and $600 \mathrm{~mm}$, the model runs smoothly, and pitching Angle changes with pavement present sine. When the pavement is volatile, i.e. wavelength of $400 \mathrm{~mm}$, model tilts at a time, and pitching Angle irregularly changes. Therefore, for the tilting active control experiment, wavelength $400 \mathrm{~mm}$ should be used. 


\section{Control system design}

\subsection{ADAMS and MATLAB hybrid modeling}

In order to control the orchard vehicle tilting actively, the MATLAB/Simulink module should be used to implant control algorithm, so as to realize the joint simulation of ADAMS and MATLAB/Simulink. The specific steps are as follows:

1) Selection and modification of the road model: orchard vehicle' movement in the irregular orchard road can be made of the vehicle response to a large number of uniform and tiny wave superposition to describe. So this paper preliminary use $2 \mathrm{~d}$ sine road as a joint simulation road.

2) Set of state variables: in order to realize the vehicle stability control, we need to give to the wheel brake signal. We definite brake signal as the input variables. Known from the analysis of the above, the input is actually vehicle's speed in the process of control. During the joint simulation, we can use controller model in Simulink to calculate the input variable values, then we pass it to the ADAMS vehicle model. Output state variables are pitching Angle and pitching Angle velocity. So the simulation model input can be as $v$, outputs are $\varphi$ and $\dot{\varphi}$.

3) Import into MATLAB/Simulink module: Use ADAMS/Control module to export the virtual prototype model. Then use MATLAB to import the model into Simulink module and set model parameters to complete the ADAMS and MATLAB hybrid modeling. The model in the MATLAB/Simulink module is as shown in figure 3.
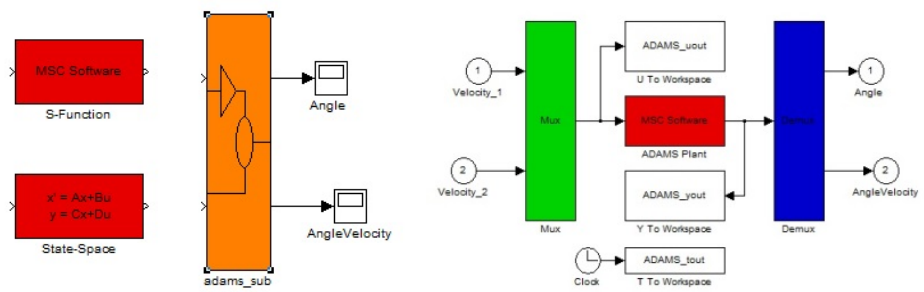

Figure.3. Simulink model

\subsection{Control model}

As shown in figure 3, adams_sub module contains the input state variable Velocity ( $v$ ) and output state variable Angle $(\varphi)$ and Angle_Velocity $(\dot{\varphi})$, parameter $v$ controlled by coefficient $K_{P}, K_{d}, \varphi$ and $\dot{\varphi}$. The working process of the active control model can be described as follows: orchard vehicle driven forward with velocity $v$ at 0 time, then encounter ups and downs road at time. According to formula (3), we get new input variable $v(t+1)$,

$$
v(t+1)=v+\Delta v
$$

By controlling $v$ with time, we can prevent orchard vehicle from tilting and control the stability of the system.

\section{Joint simulation analysis}

Through the formula (3) and the control model in figure, we can easily get when orchard vehicle driven on sine road, $\varphi$ and $\dot{\varphi}$ change. If tilt occurs, the $\varphi$ will rapidly change, so $\dot{\varphi}$ will be larger than $\varphi$. In practice, the velocity can be controlled by adjusting the $K_{P}$ and $K_{d}$, so $K_{P}$ and $K_{d}$ values have an important influence on the effectiveness of the control.

Firstly, take $K_{P}=1, K_{d}=1$. Compared with figure 8 and figure 5, it can be seen that after adding control, the simulation model go through the road safely. The $\varphi$ changes along with the road but is not stable sine curve. The change of the $v$ is a result of the combination of $\varphi$ and $\dot{\varphi}$.

In this paper, we repeated joint simulation by adjusting the value of $K_{P}$ and $K_{d}$ for many times. And chose some representative curves which is shown in figure below: 


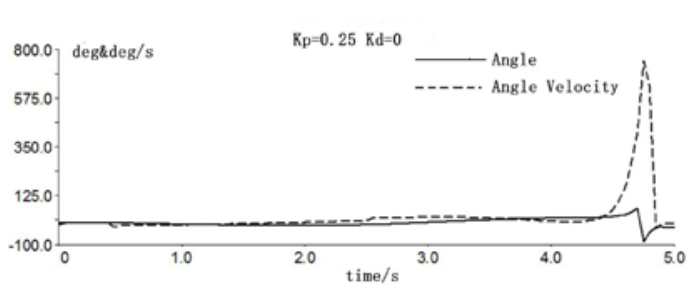

(a)

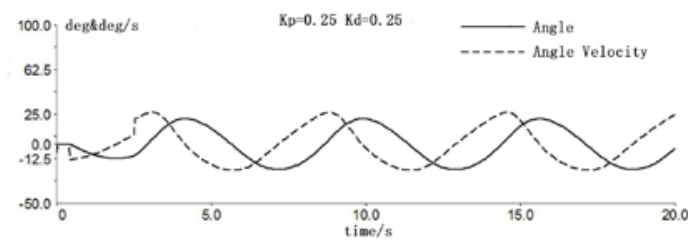

(c)

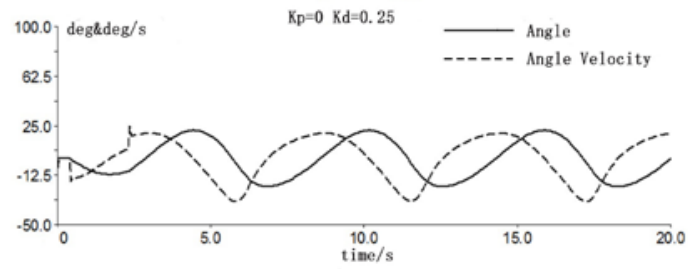

(b)

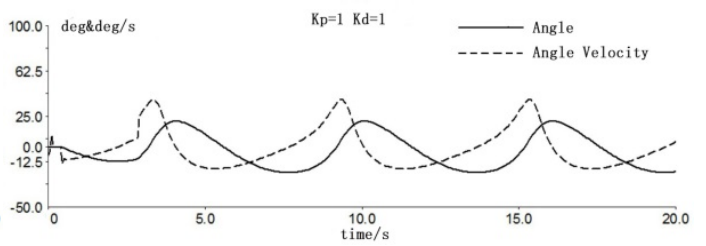

(d)

Figure.4. Simulation test results

The results shows that the control model in figure 3 effectively reduce the probability of tilt in the process of orchard vehicle driving. And we found different $K_{P}$ and $K_{d}$ values could lead to different conditions: figure 4 (a) shows that when $K_{d}=0$ and $K_{P}$ is very small, the control is too weak to effectively prevent the vehicles tilting accident; if $K_{P}=0$ and $K_{d}$ is also very small, as is shows in figure 4 (b), control model is worked although the stability of orchard vehicle is not very good. If blindly raise the value of $K_{P}$ and $K_{d}$, figure 4 (d), the speed changes really violently, which will also reduce the stability of driving. When value reaches a certain degree, $K_{P}$ and $K_{d}$ are achieved with the proper value (figure 4 (c)), not only can effectively prevent tip-over of the vehicle, and driving stability of vehicle also be improved.

\section{Conclusion}

According to the orchard vehicle driving process is not stable and easily tilt, this paper established the dynamics model of orchard vehicle and put forward a way to prevent orchard vehicle tilting to reduce the accident rate. Through the ADAMS and MATLAB joint simulation to verify the effectiveness and the feasibility of the proposed control model. At the same time, we study the effect of the adjustment coefficient of the control method. When the adjustment coefficient is too small, the control effect is not obvious, to effectively prevent tilting; when adjustment coefficient is too large, the control will cause speed ups and downs violently leading unstable driving; finally we found a suitable adjustment coefficient. The coefficient not only can effectively control the orchard vehicle tilting, and also can guarantee the stability of the drive.

\section{Acknowledgements}

This work was financially supported by National Natural Science Fund (51405492) and Chang jiang Scholars and Innovative Research Team in University of China (Grant No. IRT13039).

\section{References}

[1] Chen B, Peng H. Differential-braking-based rollover prevention for sport utility vehicles with human-in-the-loop evaluations. Journal of Biomechanics, vol. 36, no. 4-5, pp. 359-389, 2001.

[2] Preston-Thomas J, Woodroofe J H F. A Feasibility Study of a Rollover Warning Device for Heavy Trucks[C]. Transport Canada, 1990, TP 10610E.

[3] Seung-Bok Choi, Hwan-Soo Lee, Sung-Ryong Hong, et al. Control and response characteristics of a magneto-rheological fluid damper for passenger vehicle[J]. Proceedings of SPIE, 2000, 3 985:438-443.

[4] Zhang Z, Zhang N, Huang C, et al. Observer-based Hœ Control for vehicle handling and stability subject to parameter uncertainties[J]. Journal of systems and Control Engineering, 2013, 
227(9): 704-717.

[5] J P Miege, D Cebon. Active Roll Control of an Experimental Articulated Vehicle[J]. Automobile Engineering, 2003:219.

[6] FUJIOKA D D, RAUCH A, SINGHOSE W E, et al. Tip-over stability analysis of mobile boom cranes with double-pendulum payloads[C]. 2009 America Control Conference, June 10-12, 2009, St Louis, Missouri, USA, New York; IEEE, 2009: 3136-3141.

[7] Hou Baolin, Goncalves F D, Sandu C, et al. Dynamic simulation of a full vehicle with magbeto-rheological damper[C]. Proceedings of the ASME Design Engineering Division, American Society of Mechanical Engineers, New York, 2004. 